\title{
A likelihood based framework for assessing network evolution models tested on real network data
}

\author{
Richard G. Clegg \\ Department of Electrical and \\ Electronic Engineering \\ University College London \\ London, UK \\ richard@richardclegg.org
}

\author{
Raul Landa \\ Department of Electrical and \\ Electronic Engineering \\ University College London \\ London, UK \\ rlanda@ee.ucl.ac.uk \\ Miguel Rio \\ Department of Electrical and \\ Electronic Engineering \\ University College London \\ London, UK \\ m.rio@ee.ucl.ac.uk
}

\author{
Uli Harder \\ Department of Computing \\ Imperial College London \\ London, UK \\ uh@doc.ic.ac.uk
}

\begin{abstract}
This paper presents a statistically sound method for using likelihood to assess potential models of network evolution. The method is tested on data from five real networks. Data from the internet autonomous system network, from two photo sharing sites and from a co-authorship network are tested using this framework.
\end{abstract}

\section{Keywords}

Network topologies, likelihood models, network evolution

\section{INTRODUCTION}

It has been found that networks arising in very different contexts share some structural statistical properties, for example a power law in their degree distribution. Such networks include the Internet Autonomous System (AS) topology, the WWW hyperlink graph, co-authorship networks, sexual contact networks, social networks based on email exchange, biological networks and others. For examples and references see [3, table 3.1]

One common hypothesis for the basis of these shared characteristics is the presence of common elementary network development processes, such as the preferential attachment model of Barábasi et al. 2. Other models have been proposed for the evolution of specific classes of networks. Many authors have proposed models which attempt to explain the evolution of a target network in terms of simple rules which produce artificial networks with the same characteristics as a given target network. Examples of models of this kind can be found in $1,1,2,4,11$. In the literature, such models

Permission to make digital or hard copies of all or part of this work for personal or classroom use is granted without fee provided that copies are not made or distributed for profit or commercial advantage and that copies bear this notice and the full citation on the first page. To copy otherwise, to republish, to post on servers or to redistribute to lists, requires prior specific permission and/or a fee.

SIMPLEX'09, July 1, 2009, Venice, Italy.

Copyright 2009 ACM 978-1-60558-704-2/09/07 ...\$10.00. are usually tested by growing an artificial model of the same size as the target network and comparing several network statistics on the real and artificial networks.

In this paper we propose the Framework for Evolutionary Topology Analysis (FETA). This framework provides several advantages when compared to the usual method of testing models: a single likelihood based measure of how well a proposed model explains the observed network evolution, it uses network evolution data rather than a single static network snapshot, and it includes a method for creating new network models from linear combinations of sub-models and a method for optimising the mixture of these sub-models. The FETA allows the assessment of network models without growing artificial models and comparing them to the target network, making model testing much faster. This paper is a companion to [5] which introduced the framework and showed it could recover known model parameters for artificial network models. This paper shows that the FETA framework can be used to investigate a variety of real networks. The class of models which FETA can work with includes Barabási-Albert (BA) 2], Albert-Barabási (AB) 1], Generalised Linear Preference (GLP) 4] and Positive Feedback Preference (PFP) 11.

\section{A LIKELIHOOD BASED FRAMEWORK FOR ASSESSING NETWORK MODELS}

The probabilistic models used by FETA are described in terms of two components referred to as an inner model and an outer model.

DEFINITION 1. The outer model chooses the operation that transforms the current network. This could be add a node, add a link between existing nodes, delete a node or delete a link between nodes.

DEFINITION 2. The inner model chooses the entity on which the operation will act. More simply it defines a probabilistic model which gives the probabilities of choosing nodes or links for the add or remove operation selected by the outer model.

A simple example would be the AB model. This would correspond to an outer model which adds a new node and 
then chooses exactly three inner nodes to connect to it. The inner model assigns probabilities to each inner node exactly proportional to their node degree. As is common in the literature, the main focus of FETA is on the inner model. The framework is flexible enough to allow or disallow node and edge removal, non-simple and directed graphs. For this paper, however, only connected, simple, undirected graphs which never lose nodes or edges are considered.

Let $G_{0}$ be the known state of the graph at a certain time. Assume that graph is known for each time an edge is added up to some step $t\left(G_{0}, G_{1}, \ldots, G_{t}\right.$ is known). Let $\theta$ be a proposed model which attempts to explain this evolution. The model $\theta$ assigns probabilities to entities in the network at each step of the network evolution.

In order to simplify the explanation, assume that the outer model always involves the choice of a single existing node to connect to a new node. Let $C=\left(N_{1}, \ldots, N_{t}\right)$ be the ordered list of nodes selected at each step derived from $G_{0}, \ldots, G_{t}$. Let $p_{i}(j \mid \theta)$ be the probability that inner model $\theta$ assigns to node $j$ at step $i$ - that is, the probability that node $j$ is chosen at step $i$. To be a valid model $\theta$ should ensure that $\sum_{j} p_{i}(j \mid \theta)=1$ where the sum is over nodes. The following theorem can easily be shown [5].

TheOREM 1. Let $C=\left(N_{1}, \ldots, N_{t}\right)$ be the observed node choices at steps $1, \ldots, t$ of the evolution of the graph $G$. Let $\theta$ be some hypothesised valid inner model which assigns a probability $p_{j}(i \mid \theta)$ to node $i$ at step $j$. The likelihood of the observed $C$ given $\theta$ is

$$
L(C \mid \theta)=\prod_{j=1}^{t} p_{j}\left(N_{j} \mid \theta\right) .
$$

Note that the probability $p_{j}(i \mid \theta)$ may depend on many things including past history, node properties exogenous to the graph and previous node choices. As long as these are observable then the calculation is still easy to make. The next step is to define a null model $\theta_{0}$ to compare the hypothesised model $\theta$ against.

Definition 3. The null model $\theta_{0}$ is defined as the model which gives every node in the choice set equal probability (this can also be thought of as the random model). The per choice likelihood ratio $c_{0}$ is the likelihood ratio between $\theta$ and the null model $\theta_{0}$ normalised by the number of choices.

$$
c_{0}=\left[\frac{L(C \mid \theta)}{L\left(C \mid \theta_{0}\right)}\right]^{1 / t} .
$$

The quantity $c_{0}$ is one if $\theta$ is exactly as likely as $\theta_{0}$ to have given rise to the observed choices $C$. If $c_{0}$ is greater than one then $\theta$ is more likely and if less than one it is less likely. Note that two hypothesised models can be compared by looking at the ratio of their $c_{0}$ values. Note also that $c_{0}$ and $L(C \mid \theta)$ are simply different ways of looking at the model likelihood. It is also worth noting that, using the same underlying source code to calculate the probabilities, generating an artificial network model of the same size as the real target network took much longer (sometimes a hundred times as long) than measuring the likelihood statistics. Other standard statisitics such as deviance and Akakai's Information Criterion can also trivially be calcualted from $L(C \mid \theta)$.
If $\theta_{i} i=1,2, \ldots, N$ are valid models for a given network then $\theta=\sum_{i=1}^{N} \beta_{i} \theta_{i}$ with $\beta_{i} \in[0,1]$ and $\sum_{i=1}^{N} \beta_{i}=1$ is also a valid model. This allows sub models to be linearly combined to form hybrid models. The linear $\beta$ parameters and other model parameters (for example the $\delta$ in the PFP model) can be optimised to find the model which has the highest $c_{0}$ value for a given target network. Optimisation of the $\beta$ parameters can be performed using generalised linear modelling as described in [5].

Let $d_{i}$ be the degree of node $i$ and $T_{i}$ be the triangle count (the number of triangles, or 3-cycles, the node is in). The model components considered for this paper included the following: $\theta_{0}$ - the null model (random model) assumes all nodes have equal probability $p_{i}=k_{0} ; \theta_{d}$ - the degree model (preferential attachment) assumes node probability $p_{i}=k_{d} d_{i} ; \theta_{T}$ - the triangle model assumes node probability $p_{i}=k_{t} T_{i} ; \theta_{S}$ - the singleton model assumes node probability $p_{i}=k_{S}$ if $d_{i}=1$ and $p_{i}=0$ otherwise; $\theta_{D}-$ the doubleton model assumes node probability $p_{i}=k_{D}$ if $d_{i}=2$ and $p_{i}=0$ otherwise; $\theta_{R}(n)$ - the "recent" model where $p_{i}=k_{H}$ if a node was one selected in the last $n$ selections and $p_{i}=0$ otherwise and $\theta_{p}^{(\delta)}$ - the PFP model assumes node probability $p_{i}=k_{p} d_{i}^{1+\delta \log _{10}\left(d_{i}\right)}$. The $k_{\bullet}$ are all normalising constants to ensure $\sum_{i} p_{i}=1$.

So, for example $\theta=0.5 \theta_{d}+0.4 \theta_{p}(0.05)+0.1 \theta_{S}$ is a model which is $50 \%$ preferential attachment, $40 \% \mathrm{PFP}$ with $\delta=$ 0.05 and $10 \%$ singleton model.

\section{REAL DATA TESTING}

The FETA procedure is used to create inner models for several networks of interest. Section 3.1 fits models to a coauthorship network inferred from the arXiv database. Section 3.2 fits models to a view of the AS network topology referred to here as the UCLA AS network and section 3.3 fits models to a second view of the AS topology, which we refer to here as the RouteViews AS network. Section 3.4 fits network evolution models to a network derived from user browsing behaviour on a photo sharing site known as "gallery" and section 3.5 fits models to a social network derived from the popular photo sharing site Flickr. The networks are summarised below.

\begin{tabular}{|c|lll|}
\hline Network & edges & nodes & edge/node \\
\hline arXiv & 15,788 & 9,121 & 1.73 \\
UCLA AS & 93,957 & 29,032 & 3.24 \\
RouteViews AS & 94,993 & 33,804 & 2.81 \\
gallery & 50,472 & 26,958 & 1.87 \\
Flickr & 98,931 & 46,557 & 2.13 \\
\hline
\end{tabular}

For each data set, three inner models are tried: a random model, a pure PFP model (with an optimally tuned $\delta$ for connections to new nodes, and another one for internal edges) and the best model found by trying all combinations of submodels using the generalised linear model fitting procedure described in [5] and maximising the per choice likelihood ratio $c_{0}$ - separate inner models are fitted to connections from new nodes and connections between existing nodes. These models will be called, for convenience, random, PFP and best - where best here should be understood as the best possible model using combinations of the submodels consdered rather than being the best possible model of the network. Note that this model does not contain the interactive growth model from [1] and the results that fol- 
low should not be taken as a criticims of PFP as described in 11 .

Because the outer model was not the subject of interest here the outer model was simply taken to be the actual operation observed in the real data. In practice this was little different from the results obtained from the outer model derived simply by calculating empirically from the data two distributions: 1) the number of inner nodes each new node connects to on arrival, 2) the number of inner edges connected between each new node arrival. The outer model behaviour can be drawn from these distributions and the results are little changed.

For each model, $c_{0}$ from definition 3 is measured. Several network statistics are then measured for comparison. Simple statistics were chosen: $d_{1}$ is the proportion of nodes which have degree one and $d_{2}$ the proportion of nodes with degree two, max $d$ is the maximum degree of any node and $\overline{d^{2}}$ is the mean square of the node degrees (a measure of variance) note that $\bar{d}$ is not a useful measure, it is set by the outer model and would be the same for all models. The clustering coefficient $\gamma$ is a measure of the proportion of possible triangles present in the graph. The assortativity coefficient $r$ is positive when nodes attach to nodes of like degree (high degree nodes attach to each other) and negative when high degree nodes tend to attach to low degree nodes. For full definitions of all these quantities see [7].

\subsection{Fitting the arXiv data set}

A publication co-authorship network was obtained from the online academic publication network arXiv!. The first paper was added in April 1989 and papers are still being added to this day. To keep the size manageable, the network was produced just from the papers with the category label "math". The network is a co-authorship network: an edge is added when two authors first write a paper together. The author match is on first initial and surname, though it is clear this will allow some collisions. One paper ${ }^{2}$ was removed from analysis. The paper has 60 authors (far more than the next largest) which would add a distorting size 60 clique (1,732 links). The arXiv network has also been analysed by (amongst others) 8 from the perspective of growth rates and clique addition.

Obviously the random model has $c_{0}=1$. The pure PFP model has $\delta=-0.17$ and $c_{0}=1.31$. The best model has the model for connecting to new nodes $0.56 \theta_{p}(-0.29)+$ $0.28 \theta_{R}(3)+0.16 \theta_{S}(\mathrm{PFP}+$ recent + singleton $)$ and the model for connecting between existing nodes $0.57 \theta_{p}(-0.03)+$ $0.39 \theta_{R}(3)+0.04 \theta_{S}(\mathrm{PFP}+$ recent + singleton $)$ together this gives $c_{0}=6.24$. This implies that PFP should be slightly better than random and best should be better than both.

Figure 1 shows the results for the arXiv data. As can be seen, for $d_{1}$ and max $d$ the results are in the order predicted and, for the best model are a good fit to the real data. For $d_{2}$ random is slightly better than PFP. For $\overline{d^{2}} \mathrm{PFP}$ is a better fit than the best model although both are very similar and quite close to the real data. For $\gamma$ and $r$ all models are similar and similarly bad fit to the real data. No models have captured these second and third order statistics. The obvious reason for this is that uniquely in the arXiv data nodes are all added as cliques. If $n$ authors write a paper together then a clique

\footnotetext{
${ }^{1}$ http://www.arxiv.org

${ }^{2}$ http://arxiv.org/abs/math/0406190
}

of size $n$ (some nodes in which are already present on the network) is added. An obvious improvement to the model could be obtained by having "add clique of size $n$ " as an outer model operation and an inner model which selected which node(s) in the clique were already present in the network.

\subsection{UCLA AS data set}

The data set we refer to here as the UCLA AS data set is a view of the Internet AS topology seen between January 2004 and August 2008. It comes from the Internet topology collection ${ }^{3}$ maintained by Oliviera et al. [10. These topologies are updated daily using data sources such as BGP routing tables and updates from RouteViews, RIPE Abilent5 and LookingGlass servers. Each node and link is annotated with the times it was first and last observed during the measurement period. The AS data set has been analysed by several other researchers but few have analysed the data set as it grows. 6] uses linear modelling techniques to assess the goodness of fit of a preferential attachment model.

The data is preprocessed by removing all edges and nodes which are not seen in the final sixty days of the data, so that the final state of the evolution of the network is the AS network as it is in August 2008. Edges are introduced into the network in the order of their first sighting. If this would cause the network to become disconnected, their introduction is delayed. Data is available from January 2004 and a "warm up" period is given with $G_{0}$ (the starting graph) taken to occur slightly after this start date.s

For the UCLA data the best pure PFP model was with $\delta=$ 0.0015 which had $c_{0}=6.326$. The best model was, for the model to connect to new nodes $0.81 \theta_{p}(0.0015)+0.19 \theta_{R}(1)$ and for the model to connect between existing edges $0.75 \theta_{d}+$ $0.2 \theta_{R}(1)+0.05 \theta_{S}$. This model had $c_{0}=11.43$.

Figure 2 shows the results for the UCLA network. For $d_{1}, d_{2}, \max d$ and $\overline{d^{2}}$ the results are in the expected order and for all but $d_{2}$ are quite close (no model predicts $d_{2}$ very well). For assortativity, PFP is slightly better than best. For clustering coefficient no models are correct.

\subsection{RouteViews AS data set}

For the present paper we define the RouteViews AS data set as the view of the Internet AS topology from the point of view of a single RouteViews data collector. The raw data used to construct it comes from the University of Oregon Route Views Project 6 . A fuller description can be found in [5]. The best pure PFP model was $\theta_{p}(0.005)$ and the best model found which was $0.81 \theta_{p}(0.014)+0.17 \theta_{R}(1)(\mathrm{PFP}+$ "recent") to connect new nodes and $0.71 \theta_{d}+0.22 \theta_{R}(1)+$ $0.07 \theta_{S}$ (preferential attachment + "recent" + singleton) to connect edges between existing nodes. The PFP model $\theta_{p}(0.005)$ had $c_{0}=4.81$ and the best model had $c_{0}=8.06$. This suggested that best would be better than PFP which would be better than random.

For most statistics, the models were in the order expected but for $\gamma$ and $r$ PFP was slightly better than best. For $d_{1}$ and max $d$ the PFP model was little different to random although in the case of max $d$, random predicted unrealistically slow growth. Overall, however, the model order was that predicted by the $c_{0}$ values.

\footnotetext{
${ }^{3}$ http://irl.cs.ucla.edu/topology/

${ }^{4}$ http://www.ripe.net/db/irr.html/

5 http://abilene.internet2.edu/

6 http://www.routeviews.org
} 

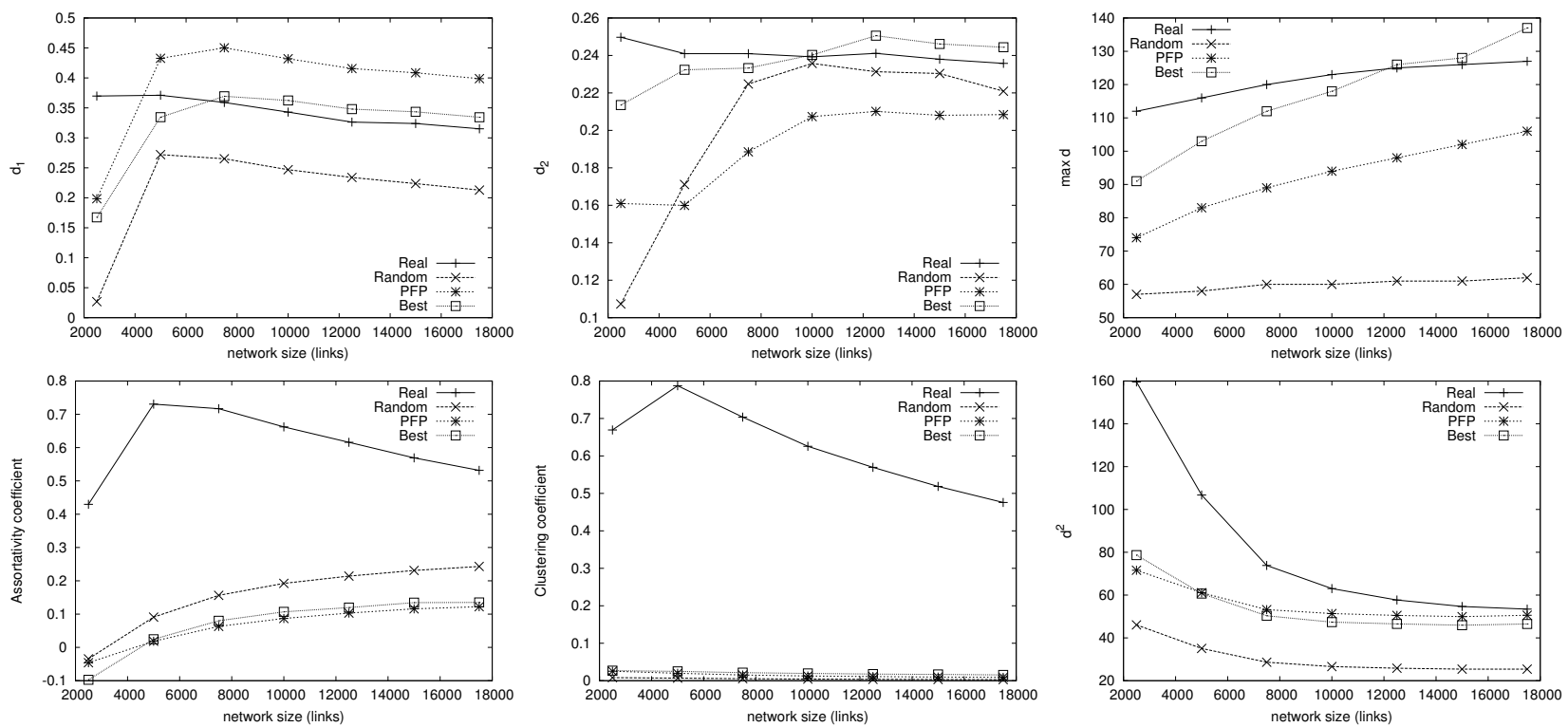

Figure 1: Results for arXiv network
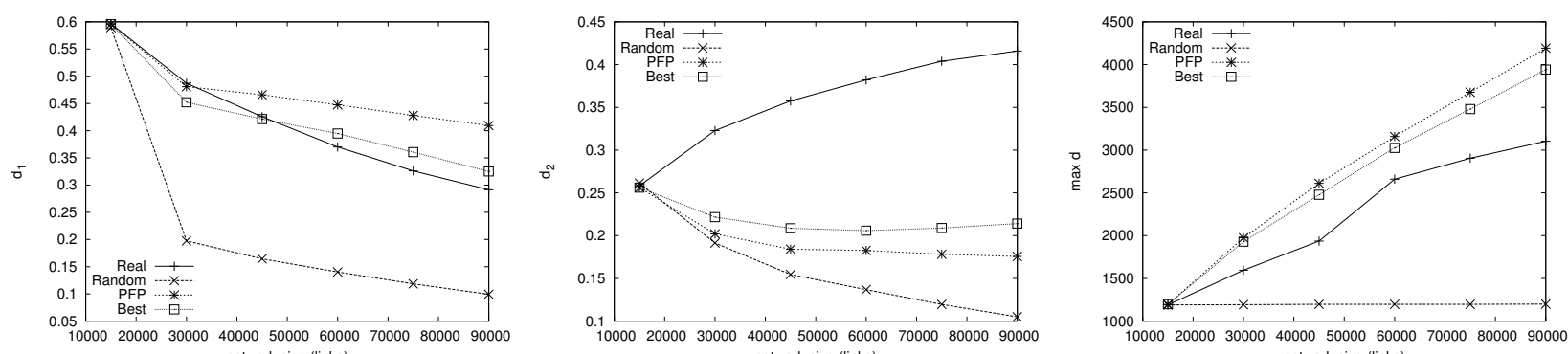
network size (links)
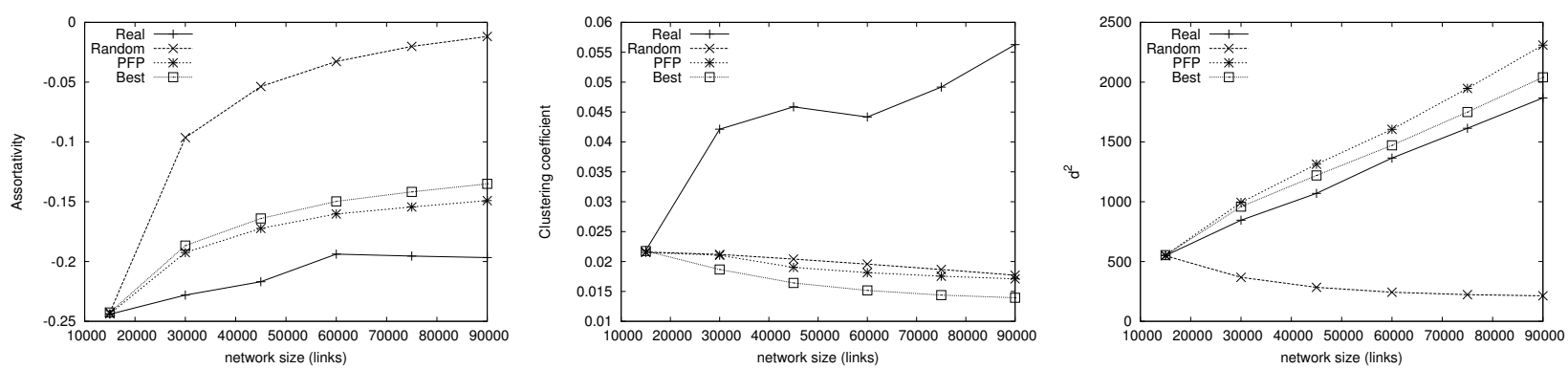

Figure 2: Results for UCLA network

\subsection{Fitting the gallery data set}

The website known simply as "gallery' 17 is a photo sharing website. To be able to upload pictures and have some control over the display of pictures, users have to create an account and login. From webserver logs, the path logged in users browse as they move across the network can be followed. Thus, images become nodes in the networks, and a user browsing between two photos creates a link between the two nodes that represent them. These links are overlaid for all users in order to form the network analysed here.

\footnotetext{
${ }^{7}$ http://gallery.future-i.com/
}

The best pure PFP model for the gallery data was with $\delta=-0.4$, however, unusually, this model was worse than random with $c_{0}=0.8515$. The best model had, for its connections to new nodes, $0.57 \theta_{S}+0.24 \theta_{d}+0.19 \theta_{R}(3)$ (singleton + preferential attachment plus "recent") and for its connections between existing nodes $0.61 \theta_{p}(-0.05)+0.39 \theta_{R}(5)$. This model had a per choice likelihood ratio $c_{0}=12.93$.

Figure 3 shows the results for the gallery network. From the $c_{0}$ values we would expect random to actually be slightly better than PFP and best to be much better than either. This order is followed for $d_{1}, d_{2}$ and $\overline{d^{2}}$ and seems to be for $\gamma$ although all models are incorrect here. For assortativity 

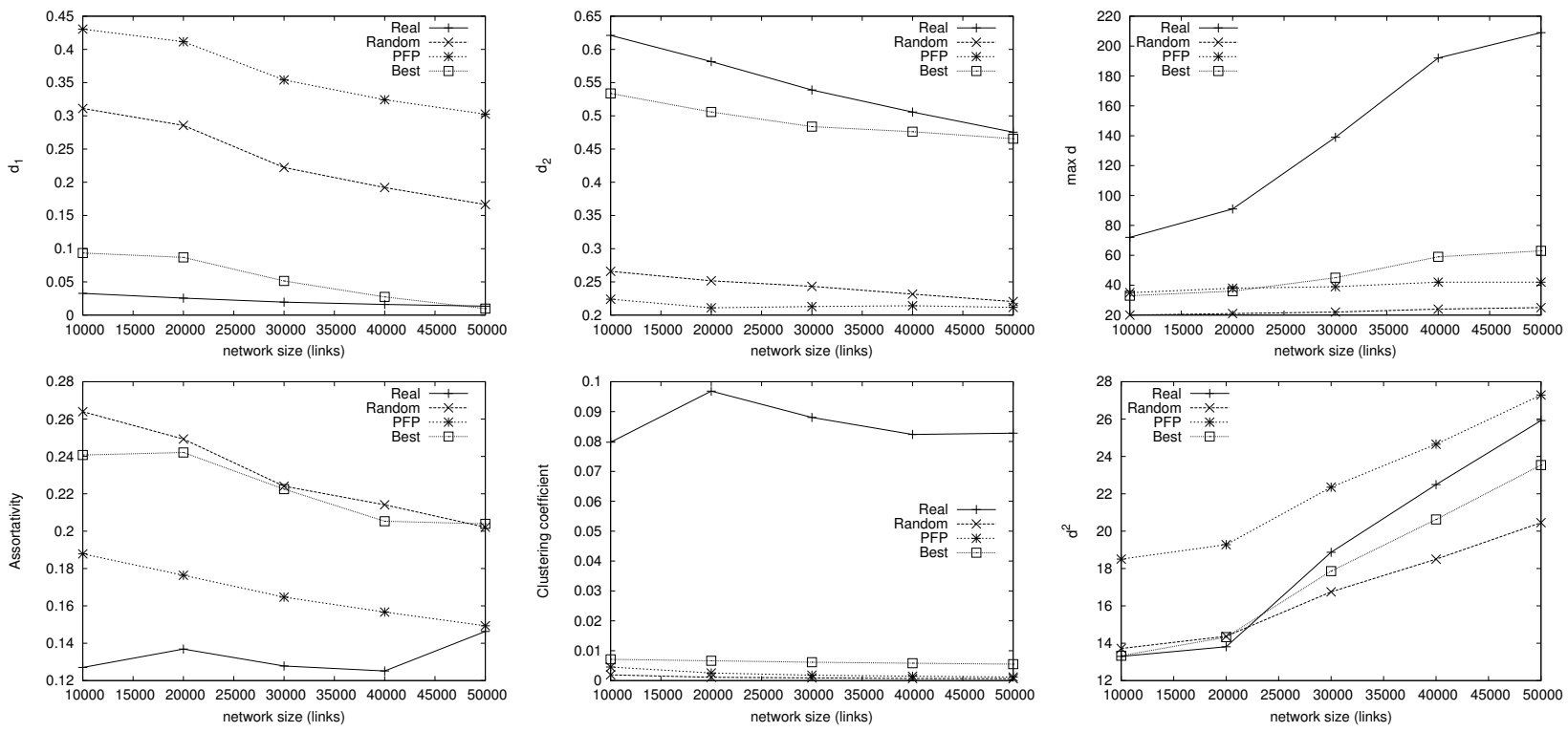

Figure 3: Results for gallery network

$r$ PFP is unexpectedly the best model and for max $d$ it is better than random. In all cases apart from $r$ the best model is closest to the real data. Again the $c_{0}$ statistic seems to be a good reflection of the closeness of network statistics, particularly "first order" statistics.

\subsection{Fitting the Flickr data set}

The Flickl 8 website allows users to associate themselves with other users by naming them as Contacts. In 9 the authors describe how they collected data for the graph made by users as they connect to other users. The first 100,000 links of this network are analysed here. The graph is generated by a web-crawling spider so the order of arrival of edges is the order in which the spider moves between the users rather than the order in which the users made the connections. Thus, the evolution dynamics of this network will be determined in part by the spidering code.

The best pure PFP model for the Flickr data was with $\delta=$ 0.015 and this had $c_{0}=28.29$. The best model had as the model for new node connections simply $0.99 \theta_{R}(1)+0.01 \theta_{d}$ and for connections between existing nodes the best model was $0.52 \theta_{p}(-0.22)+0.48 \theta_{R}(1)$. This model had the very high per choice likelihood ratio of $c_{0}=430.5$ - this is because the new node model is almost entirely deterministic, new nodes follow by browsing from old nodes. It is because the network was from a browsing pattern that gave the high proportion of $\theta_{R}(1)$ "recent" especially in the new node model.

Figure 4 shows the results for the Flickr network. From the $c_{0}$ values we would expect the best model to be much better than the PFP model which is in turn much better than the random model. In fact this is not reflected as strongly in the statistics as in the previous modelling. For $d_{1}, d_{2}$ and $r$ the statistics are as expected and best is relatively close. For $\gamma$, PFP is worse not better than random. For max $d$ and $\overline{d^{2}}$ no models are good and the order is not that predicted - PFP is slightly better than best. This may be due

\footnotetext{
8 http://flickr.com/
}

to the presence of a single extremely high degree node (degree 11,053 when the network has only 46,557 nodes) more than ten percent of the links in the network are to this single node.

\subsection{Discussion of model fitting}

In general the FETA model assessment performed extremely well in these tests. The models were fitted solely with regard to the likelihood value, without measuring network statistics in advance. In all cases, we believe an impartial observer would rank the models in the same order as the $c_{0}$ values. FETA was much faster than growing and testing many models. A GLM (generalised linear model) procedure as described in 5 allows optimisation of linear parameters and dozens of potential sub model combinations can be tested in the space of an hour or so. Growing artificial networks and testing network statistics can take longer than this to assess a single model. The submodels used focused on first degree node properties (mainly degree) and this may explain why $\gamma$ and $r$ were not always well fitted.

Some common observations can be made about the models fitted. PFP and "recent" were the most commonly used model components. As expected, PFP models had a negative $\delta$ (sublinear growth) when the node might be subject to overloading (an author can only author so many papers a Flickr user can only have time for a certain number of friends) but positive in systems where no such overloading was likely (an AS will become more efficient at adding connections as more people add them). The "spidering" nature of the Flickr data produced an unusual model for new node connections which were almost always connected from the most recently connected node, this makes sense in a "crawling" environment. (The likelihood of $\theta_{R}(1)$ on its own was zero since at least once this was not the behaviour observed). The two AS data sets ended up with quite similar models which is extremely encouraging as the fitting was done independently. 

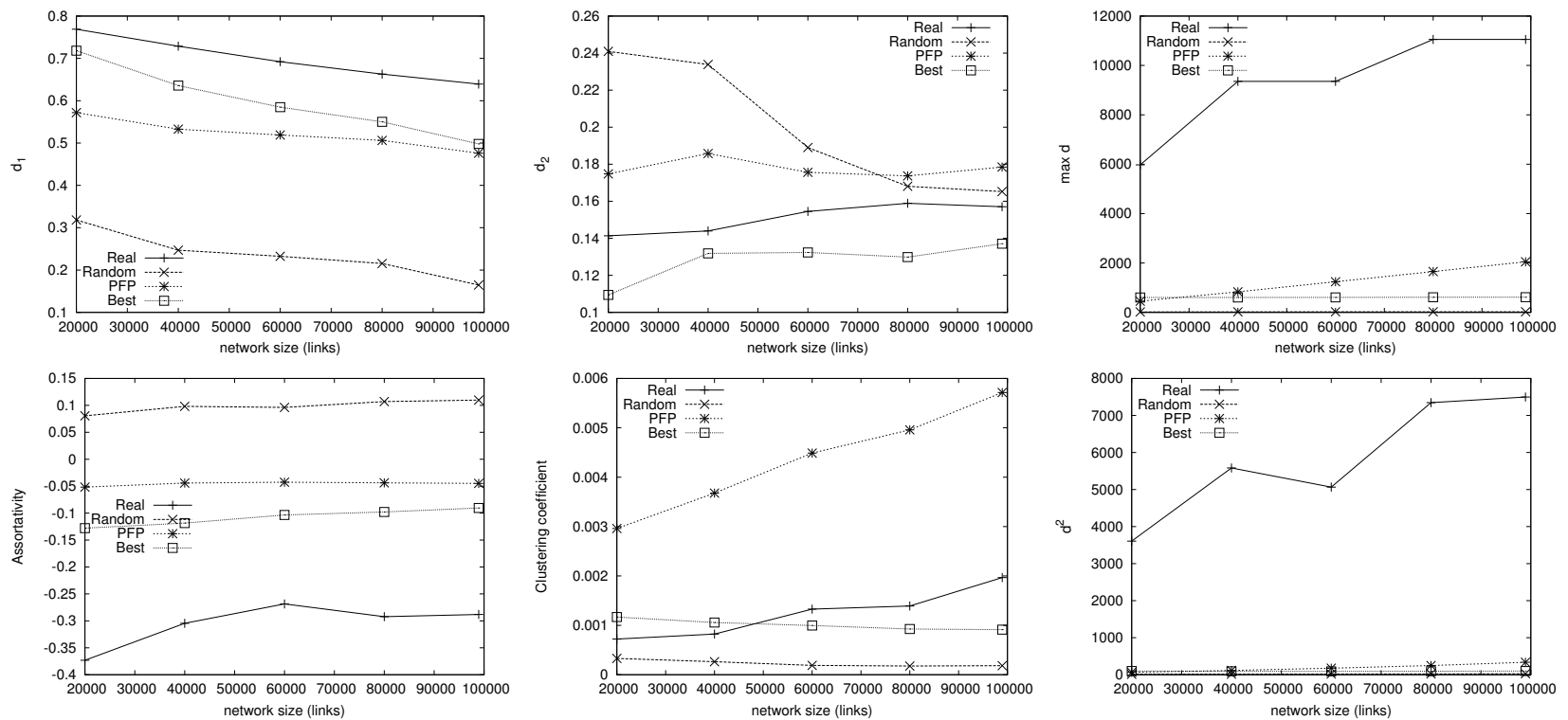

Figure 4: Results for Flickr network

\section{CONCLUSIONS}

The FETA framework demonstrated in this paper is an excellent way to test hypothesised models of network evolution if the data set allows this (evolutionary data must be available). In the tests here the model likelihood $c_{0}$ was an excellent predictor of how close network statistics would be to those same statistics measured on the target network. In addition the statistics usually behaved in the same way as the network evolved. The framework has proved a useful tool for the investigation of five real target networks.

The model components here did a reasonable but far from perfect job of replicating the real model statistics. However, the aim of the paper was to show that the framework could assess models not to design perfect models. In this case the models most common failure was failure to replicate clustering coefficient and assortativity. This is perhaps inevitable as the models were built from components which relied on first order statistics. Altering the inner model to include second order statistics or altering the outer model (for example to allow addition of cliques) could improve this behaviour.

Overall though, the FETA framework is an advance in assessment of network topology models. It accounts for the evolution of the network rather than trying to match a static snapshot. It provides a single statistically rigorous likelihood for a model rather than relying on trying to match a large number of possibly correlated statistics. It is computationally cheaper than growing an artificial test network and measuring statistics to compare with the target network.

Much remains to be done with FETA to improve it. The outer model needs attention next and it seems that a similar likelihood procedure would prove successful here. Many different sub models can be tried, in particular focussing on second and higher order statistics seems important. The authors welcome collaboration and all software and data used here can be found at http://www.richardclegg.org/software/FETA

\section{REFERENCES}

[1] R. Albert and A.-L. Barábasi. Topology of evolving networks: local events and universality. Physical Review Letters, 85:5234, 2000.

[2] A.-L. Barabási and R. Albert. Emergence of scaling in random networks. Science, 286(5439):509-512, (1999).

[3] S. Bornholdt and H. G. Schuster, editors. Handbook of Graphs and Networks. Wiley, 2003.

[4] T. Bu and D. Towsley. On distinguishing between Internet power law topology generators. In Proceedings of IEEE INFOCOM, New York, NY, June 2002.

[5] R. G. Clegg, R. Landa, H. Haddadi, and M. Rio. Measuring the likelihood of models for network evolution. In IEEE INFOCOM Workshop NetSciCom, 2009.

[6] A. Dhamdhere and C. Dovrolis. Ten years in the evolution of the Internet ecosystem. In Proc. of the ACM SIGCOMM Internet Meas. Conf. (IMC), 2008.

[7] H. Haddadi, G. Iannaccone, A. Moore, R. Mortier, and M. Rio. Network topologies: Inference, modelling and generation. IEEE Comm. Surveys and Tutorials, 10(2), 2008.

[8] J. Leskovec, J. Kleinberg, and C. Faloutsos. Graphs over time: Densification laws, shrinking diameters and possible explanations. In Proc. 11th ACM SIGKDD Intl. Conf. on Know. Disc. and Data Mining, 2005.

[9] A. Mislove, H. S. Koppula, K. P. Gummadi, P. Druschel, and B. Bhattacharjee. Growth of the Flickr social network. In WOSP '08: Proceedings of the first workshop on Online social networks, pages 25-30. ACM, 2008.

[10] R. Oliveira, B. Zhang, and L. Zhang. Observing the evolution of Internet AS topology. In Proceedings of ACM SIGCOMM, Kyoto, Japan, 2007.

[11] S. Zhou and R. J. Mondragón. Accurately modeling the Internet topology. Phys. Rev. E, 70(066108):1-7, 
2004. 